\title{
Genome-wide analysis of circular RNAs in goat skin fibroblast cells in response to Orf virus infection
}

\author{
Feng Pang ${ }^{1}$, Mengmeng Zhang ${ }^{1}$, Xiaojian Yang ${ }^{1}$, Guohua Li ${ }^{1}$, Shu Zhu ${ }^{1}$, Xin Nie ${ }^{1}$, Ruiyong Cao ${ }^{1}$, \\ Xiaohong Yang ${ }^{1}$, Zhenxing Zhang ${ }^{1}$, Haifeng Huang ${ }^{1}$, Baobao Li ${ }^{1}$, Chengqiang Wang ${ }^{1}$, Li Du ${ }^{1}$, Fengyang \\ Wang Corresp. 1 \\ ${ }^{1}$ College of Animal Science and Technology, College of Tropical Agriculture and Forestry, Hainan University, Hainan Key Lab of Tropical Animal
Reproduction \& Breeding and Epidemic Disease Research, Hainan University, Haikou, China
} Corresponding Author: Fengyang Wang

Email address: fywang68@163.com

Orf, caused by Orf virus (ORFV), is a globally distributed zoonotic disease responsible for serious economic losses in the agricultural sector. However, the mechanism underlying ORFV infection remains largely unknown. Circular RNAs (circRNAs), a novel type of endogenous non-coding RNAs, play important roles in various pathological processes but their involvement in ORFV infection and host response is unclear. In the current study, whole transcriptome sequencing and small RNA sequencing were performed in ORFVinfected goat skin fibroblast cells and uninfected cells. A total of 151 circRNAs, 341 messenger RNAs (mRNAs), and 56 microRNAs (miRNAs) were differently expressed following ORFV infection. Four circRNAs: circRNA1001, circRNA1684, circRNA3127 and circRNA7880 were validated by qRT-PCR and Sanger sequencing. Gene ontology (GO) analysis indicated that host genes of differently expressed circRNAs were significantly enriched in regulation of inflammatory response, epithelial structure maintenance, positive regulation of cell migration, positive regulation of ubiquitin-protein transferase activity, regulation of ion transmembrane transport, etc. The constructed circRNA-miRNA-mRNA network suggested that circRNAs may function as miRNA sponges indirectly regulating gene expression following ORFV infection. Our study presented the first comprehensive profiles of circRNAs in response to ORFV infection, thus providing new clues for the mechanisms of interactions between ORFV and the host. 
1 Genome-wide analysis of circular RNAs in Goat skin

2 fibroblast cells in response to Orf virus infection

3 Feng Pang, Mengmeng Zhang, Xiaojian Yang, Guohua Li, Shu Zhu, Xin Nie, Ruiyong Cao,

4 Xiaohong Yang, Zhenxing Zhang, Haifeng Huang, Baobao Li, Chengqiang Wang, Li Du,

$5 \quad$ Fengyang Wang *

6 College of Animal Science and Technology, College of Tropical Agriculture and Forestry, Hainan

7 University, Hainan Key Lab of Tropical Animal Reproduction \& Breeding and Epidemic Disease

8 Research, Haidian Island, Haikou 570228, People's Republic of China

$9 *$ Correspondence: fywang68@163.com

Tel.: +8613648616818

Abstract: Orf, caused by Orf virus (ORFV), is a globally distributed zoonotic disease responsible for serious economic losses in the agricultural sector. However, the mechanism underlying ORFV infection remains largely unknown. Circular RNAs (circRNAs), a novel type of endogenous noncoding RNAs, play important roles in various pathological processes but their involvement in ORFV infection and host response is unclear. In the current study, whole transcriptome sequencing and small RNA sequencing were performed in ORFV-infected goat skin fibroblast cells and uninfected cells. A total of 151 circRNAs, 341 messenger RNAs (mRNAs), and 56 microRNAs (miRNAs) were differentially expressed following ORFV infection. Four circRNAs: circRNA1001, circRNA1684, circRNA3127 and circRNA7880 were validated by qRT-PCR and Sanger sequencing. Gene ontology (GO) analysis indicated that host genes of differentially expressed circRNAs were significantly enriched in regulation of inflammatory response, epithelial structure maintenance, positive regulation of cell migration, positive regulation of ubiquitinprotein transferase activity, regulation of ion transmembrane transport, etc. The constructed circRNA-miRNA-mRNA network suggested that circRNAs may function as miRNA sponges indirectly regulating gene expression following ORFV infection. Our study presented the first comprehensive profiles of circRNAs in response to ORFV infection, thus providing new clues for the mechanisms of interactions between ORFV and the host.

\section{Introduction}

Circular RNAs (circRNAs), a new member of non-coding RNAs, are generally produced by back-splicing of pre-mRNA(Barrett \& Salzman 2016; Barrett et al. 2015). Because of their unique circular structure, circRNAs can resist the activity of RNA exonuclease digestion. Thus, they are more stable and have longer half-lives than their linear transcripts in vivo (Jeck et al. 2013; Suzuki et al. 2006). Moreover, circRNAs are highly abundant and are conserved among a variety of species (Barrett \& Salzman 2016; Memczak et al. 2013). A number of circRNAs also present cell type- and tissue-specific expression patterns (Salzman et al. 2013; Szabo et al. 2015).

Increasing evidence suggests that circRNAs can function as competing endogenous RNAs (ceRNAs) or miRNA sponges, thereby acting as post-transcriptional regulatory elements (Hansen et al. 2013; Qu et al. 2015; Zhang et al. 2016). For instance, the circRNA ciRS-7, containing 73 conventional miR-7 binding sites, strongly inhibits miR-7 activity, thus increasing the expression 
levels of miR-7 targets (Hansen et al. 2013). Many studies show that circRNAs play key roles in the progression of several serious types of disease such as cancer (Li et al.2015; He et al. 2017a; Tian et al. 2017). Moreover, circRNAs are associated with viral infection and host-pathogen interactions. Shi et al. performed circRNA sequencing to study the expression profiles of circRNAs in Vero cells following infection with SV40 virus (Shi et al. 2017) while He et al. analyzed the roles of circRNA in host-grass carp reovirus interactions via deep sequencing (He et al. 2017b). However, the functions of circRNAs within host cells in response to Orf virus (ORFV) infection has not been studied thus far.

Orf, also called contagious ecthyma, is a globally-distributed zoonotic disease responsible for serious economic losses in the agricultural and animal husbandry industries (Friederichs et al. 2014). Infection usually occurs in the lips, oral mucosa, and around the nostrils of goats and sheep, with typical symptoms including erythema, papula, pustules, and scabs(Delhon et al. 2004). ORFV, the causative agent of Orf, is a zoonotic virus belonging to the Parapoxvirus genus (Zhang et al. 2014a). As such, humans may become infected with ORFV after coming into contact with infected animals (Kumar et al. 2014; Peng et al. 2016; Rajkomar et al. 2016). ORFV has a linear, double-stranded DNA genome approximately $130-140 \mathrm{~kb}$ in length and containing a putative 131 open reading frames (Delhon et al. 2004; Martins et al. 2017; Zhang et al. 2014a). To better understand the pathogenesis of ORFV, several previous studies have conducted genome sequencing of different ORFV isolates (Chi et al. 2015; Delhon et al. 2004; Zhao et al. 2010), as well as transcriptome sequencing of host cells following ORFV infection (Chen et al. 2017; Jia et al. 2017).

However, the specific characteristics and roles of circRNAs during ORFV infection remain unclear. Therefore, in the current study we conducted circRNA sequencing and small RNA sequencing of uninfected and ORFV-infected goat skin fibroblast (GSF) cells in an attempt to gain new insights into ORFV pathogenesis.

\section{Materials and methods}

\section{Cell culture and viral infection}

Goat skin fibroblast cells (GSF cells) were obtained from the Cell Bank of the Chinese Academy of Science (Kunming, China) and maintained in Dulbecco's modified Eagle medium (DMEM; Invitrogen, Carlsbad, CA, USA) supplemented with 10\% fetal bovine serum (Invitrogen) at $37^{\circ} \mathrm{C}$ and $5 \% \mathrm{CO}_{2}$. When GSF cells seeded in $10-\mathrm{cm}$ diameter dish reached $\sim 90 \%$ confluence, the medium was removed and cells were rinsed three times with PBS. Cells were then infected with ORFV strain JLSY (tissue culture infectious dose $50 \%=10^{6.2} / \mathrm{mL}$ ), a gift from Professor Guixue Hu and Research Associate Hongze Shao, at a multiplicity of infection of one. Following incubation for $1 \mathrm{~h}$ at $37^{\circ} \mathrm{C}$, the virus suspension was removed and cells were cultured for a further $6 \mathrm{~h}$ in standard medium.

\section{$R N A$ extraction, library construction, and sequencing}

Total RNA from both ORFV infected GSF samples (OV) and uninfected GSF samples were isolated using an Ambion mirVana miRNA Isolation Kit (Thermo Fisher Scientific, Waltham, MA, USA). The quality of total RNA were analyzed by Bioanalyzer 2100 (Agilent Technologies, Santa Clara, CA, USA), and the concentration of the total RNA was quantified using a NanoDrop 2000 (Thermo Fisher Scientific, Lafayette, Colorado, USA). In this study, circRNA libraries were constructed as below: Ribosomal RNA was removed from $5 \mu \mathrm{g}$ aliquots of total RNA using an 
Epicentre Ribo-Zero Gold Kit (Illumina, San Diego, CA, USA). Then, the RNA fractions were fragmented and were reverse transcribed using an mRNA-Seq Sample Preparation Kit (Illumina, San Diego, CA, USA). The cDNA libraries were then paired-end sequenced using an Illumina HiSeq 4000 platform (Lc-bio, Hangzhou, China). The raw and processed data have been deposited into the Gene Expression Omnibus database (https://www.ncbi.nlm.nih.gov/geo/) under accession number GSE121725. (2) Small RNA libraries: About $1 \mu \mathrm{g}$ total RNA of each sample was used for cDNA library construction with the TruSeq Small RNA Preparation Kit (Illumina, San Diego, CA, USA) following the manufacture's protocol. Then, the cDNA libraries were single-end 50bp (SE50) sequenced with an Illumina HiSeq 2500 platform (Lc-bio, Hangzhou, China). The raw and processed data have been deposited into the Gene Expression Omnibus database (https://www.ncbi.nlm.nih.gov/geo/) under accession number GSE121726.

\section{Identification and differential expression analysis of circR $N A s$ and $m R N A s$, and miRNAs}

Firstly, Cutadapt (Martin 2011) was utilized to remove reads containing undetermined bases, adaptors, and low quality bases. Then sequence quality was verified using FastQC (http://www.bioinformatics.babraham.ac.uk/projects/fastqc/). Bowtie2 (Langmead \& Salzberg 2012) and Tophat2 (Kim et al. 2013) were used to map reads to the Capra hircus (goat) reference genome (RefSeq assembly accession: GCF_001704415.1). The matched reads of each sample were assembled using StringTie (Pertea et al. 2015). StringTie and Ballgown (Frazee et al. 2015) were utilized to evaluate the expression levels of all transcripts by Fragments per kilobase per million reads (FPKM). The dysregulated mRNAs were selected with $\mid \log _{2}$ (fold change) $\mid \geq 1$ and $p \leq 0.05$ by $\mathrm{R}$ package Ballgown (Frazee et al. 2015).

Any unmapped reads were individually mapped to the goat reference genome by TopHatFusion (Kim \& Salzberg 2011). Then, reads mapped to the goat genome using TopHat-Fusion were analyzed by CIRCexplorer to identify candidate circRNAs (Zhang et al. 2016; Zhang et al. 2014b). The criteria were as follows: 1) GU/AG must occur at both ends of splice sites; 2) less than two mismatches; 3 ) more than one back-spliced junction read in at least one sample of GSF or OV group; 4) two splice sites are no more than $100 \mathrm{~kb}$ apart on the genome. The expression of circRNAs was calculated by the number of reads spanning back-splicing junction and FPKM was used to normalize the expression level of circRNAs. CircRNAs with $\mid \log _{2}$ (fold change) $\mid \geq 1$ and $p$ $\leq 0.05$ were regarded as differentially expressed by R package-edgeR (Robinson et al. 2014).

For miRNA analysis, ACGT101-miR (LC Sciences, Houston, Texas, USA) was used to acquire clean reads. Then, unique sequences containing 18 to 26 nucleotides were mapped to miRBase 21.0 by BLAST search to identify known and novel miRNAs. The expression level of miRNAs was normalized based on the read counts to tags per million counts (TPM). The significance standard was $p \leq 0.05$.

\section{Gene ontology (GO) and Kyoto Encyclopedia of Genes and Genomes (KEGG) analysis}

GO analysis was used to determine significantly enriched GO terms $(p \leq 0.05)$ by hypergeometric test ( Beißbarth \& Speed 2004; Consortium 2004). KEGG pathway analysis was used to explore significantly enriched pathways $(p \leq 0.05)$ by hypergeometric test (Kanehisa et al. 2017; Kanehisa et al. 2012). 


$$
\mathrm{P}=1-\sum_{i=0}^{\mathrm{m}-1\left(\begin{array}{c}
M \\
i
\end{array}\right)\left(\begin{array}{c}
N-M \\
m-i
\end{array}\right)}
$$

$\mathrm{N}$ : Total number of circRNA-hosting genes; $n$ : The number of circRNA-hosting genes with differential expression; M: The number of circRNA-hosting genes annotated to the GO term; $\mathrm{m}$ : The number of circRNA-hosting genes with differential expression annotated to the GO term

\section{Bioinformatics analysis and ceRNA network construction}

The circRNA-miRNA and miRNA-mRNA interactions were predicted using TargetScan 7.0 and miRanda software. TargetScan 7.0 predicts the targets of miRNAs based on seed region homologies (Agarwal et al. 2015) while MiRanda is mainly based on the combination of free energy generated by miRNAs binding to their target genes (Betel et al. 2008). The lower the free energy, the stronger the binding. TargetScan score percentiles $\geq 50$, Miranda max free energy values $<-10$ and Miranda score $>140$ were defined as the cutoff points for targets prediction. To further explore the functional role of circRNAs, a ceRNA network was constructed using Cytoscape 3.6.0 software (Shannon et al. 2003).

\section{Validation of miRNAs}

Six differentially expressed novel miRNAs (PC-3p-8215_174, PC-5p-406_14064, PC-5p2253_1210, PC-5p-5127_361, PC-3p-10316_124 and PC-3p-4306_468) with mean TPM $>30$ in GSF samples or OV samples were selected for qPCR validation. First, the total RNA was reversetranscribed using a miRNA 1st Strand cDNA Synthesis kit (Vazyme, Nanjing, China) with specific stem-loop primers (Table S1). Then, qRT-PCR assays were performed using miRNA Universal SYBR qPCR Master Mix (Vazyme, Nanjing, China) with specific forward primers (F) and the universal reverse primer (Table S1) with an ABI 7500 Real-Time PCR System (Applied Biosystems, Foster City, CA, USA). U6 snRNA was used as an internal control for normalization of the expression level of these miRNAs. All experiments were conducted independently three times.

\section{Validation of circRNAs by qRT-PCR and Sanger sequencing}

Differentially expressed circRNAs with mean FPKM $\geq 10$ (relatively high expression ) in OV samples or GSF samples as well as possessing more than one back-spliced read in at least two replicates of OV samples or GSF samples were selected for confirmation by qRT-PCR. Thus, six down-regulated circRNAs: circRNA998, circRNA1000, circRNA1001, circRNA1684, circRNA3127, circRNA4287 and three up-regulated circRNAs: circRNA5112, circRNA7880, circRNA8565 were obtained. First, total RNA was reversely transcribed to cDNA using a Revert Aid First Strand cDNA Synthesis Kit (Thermo Fisher Scientific). Next, qPCR assays were performed with divergent primers using the ABI 7500 Real-Time PCR System (Applied Biosystems, Foster City, CA, USA) with $2 \times$ SYBR qPCR Mix (Aidlab, Beijing, China). $2-\Delta \Delta C t$ method was used to calculate the relative expression level of circRNAs with goat glyceraldehyde3-phosphate dehydrogenase $(G A P D H)$ serving as an internal control. All experiments were conducted in triplicate. The PCR products from cDNA samples were ligated into pMD-19T (Takara, Dalian, China) for Sanger sequencing to determine the back-splicing junctions.

Moreover, genomic DNA(gDNA) was extracted from GSF cells using a Blood/Cell/Tissue 
163 Genome DNA Extraction Kit (Tiangen, Beijing, China). Both cDNA and gDNA were used as 164 templates for PCR amplification using specific convergent and divergent primers (Table 1). The 165 PCR products were examined using $1.5 \%$ agarose gel.

166

167

168

169

170

171

172

173

174

175

176

177

178

179

180

181

182

183

184

185

186

187

188

189

190

191

192

193

194

195

196

197

198

199

200

201

202

203

\section{Statistical analysis}

Statistical significance analysis was performed by Student's t-test, and $p \leq 0.05$ was considered statistically significant.

\section{Results}

\section{Properties of circRNAs in ORFV-infected and uninfected GSF cells}

We first performed circRNA sequencing, using the ribosomal RNA-depleted method, of three uninfected GSF samples (GSF samples) and three ORFV-infected samples (OV samples) on the Illumina HiSeq 4000 platform. We acquired an average of 86 million and 93 million raw reads for the GSF and OV groups, respectively. Clean reads, which accounted for $>98.5 \%$ of the raw reads, were obtained after the removal of the low quality raw reads. The Q30 of each sample was $\sim 93 \%$. Most reads $(\sim 85 \%)$ were linearly mapped to the goat reference genome. Among the remaining unmapped reads, approximately $1 \%$ of reads from each sample were identified as back-spliced junction reads. Furthermore, we aligned the reads unmapped to goat genome from each sample to Orf virus reference genome (Orf virus NA1/11 strain under GenBank No. KF234407.1). The results indicated that a total of 125,619 reads, 165,878 reads and 157,779 reads were mapped to Orf virus genome in OV-1, OV-2, and OV-3 sample, respectively. There were nearly zero reads aligned to Orf virus genome in uninfected GSF samples (Table 2).

Finally, 9,979 and 10,844 circRNAs with more than one back-spliced read existed in at least one sample of GSF or OV group were identified, respectively (Table S2). Among these circRNAs, 4,649 were shared between the groups, while 5,330 and 6,195 circRNAs were uniquely expressed in the GSF and OV samples, respectively (Figure 1A). Interestingly, approximately $98 \%$ of the circRNAs were exonic circRNAs (ecircRNAs), while the remaining $2 \%$ were circular intronic RNAs (ciRNA) based on their location in the goat genome (Figure 1B). These candidate circRNAs were widely distributed from chromosome 1 to chromosome 29 (Figure 1C). In particular, chromosomes 1, 2, 3, 10, and 11 produced more than 800 circRNAs, respectively. The lengths of the circRNAs varied from 300-1,300 bp (Figure 1D). More than 2,000 genes produced only one circRNA isoform, while 889 genes produced two different isoforms, 554 genes produced three circRNA isoforms, and 364 genes produced four isoforms, with the number of genes decreasing with the number of isoforms produced (Figure 1E).

Additionally, an average of 40,882 transcripts were detected in GSF samples while 40,778 transcripts were identified in three OV samples, respectively. Following miRNA sequencing, 695 mature miRNAs in GSF samples and 674 miRNAs in OV samples were acquired, respectively. The mapping overview of miRNA sequencing is provided in Table S3.

\section{Differential expression analysis of circRNAs, mRNAs and miRNAs}

Based on the filtering criteria in materials and methods, a total of 151 circRNAs were identified as differentially expressed in ORFV-infected samples compared with GSF samples. Of these, 59 circRNAs were up-regulated while 92 circRNAs were down-regulated (Figure 2A, 2B, Table S4). There were 341 differentially expressed mRNAs with 187 up-regulated mRNAs and 
154 down-regulated mRNAs. The significant differences in transcript expression between OV samples and GSF samples was presented by clustered heatmap and volcano plot (Figure 2D, 2E, Table S5). As for miRNA, 23 miRNAs were up-regulated while 33 miRNAs were down-regulated. Among the 56 differentially expressed miRNAs, 26 miRNAs were goat-derived in miRBase 21.0 and 7 miRNAs (PC-3p-8215_174, PC-5p-406_14064, PC-5p-2253_1210, PC-5p-5127_361, PC3p-10316_124, PC-3p-19472_48, PC-3p-4306_468) were first reported (Figure 2A, 2C, Table S6).

\section{GO and KEGG analysis for host genes of differentially expressed circRNAs}

Due to the fact that the biological functions of circRNAs may be associated with their corresponding parental transcripts, GO and KEGG analyses for host genes of dysregulated circRNAs were conducted in the study. The top $20 \mathrm{GO}$ terms significantly enriched $(p \leq 0.05)$ in molecular function, cellular component, and biological process are presented in Figure 3A. The top six enriched GO terms in cellular component were proteinaceous extracellular matrix, intercalated disc, voltage-gated sodium channel complex, plasma membrane, T-tubule and extracellular region. The top six enriched GO terms in molecular function were growth factor binding, voltage-gated sodium channel activity, neuropilin binding, semaphorin receptor binding, chemorepellent activity and integrin binding. Moreover, regulation of inflammatory response, epithelial structure maintenance, negative regulation of insulin secretion, positive regulation of cell migration, positive regulation of ubiquitin-protein transferase activity, regulation of ion transmembrane transport were significantly enriched in the biological process subgroup (Figure 3A, Table S7).

Next, we conducted KEGG enrichment analysis for circRNA-hosting genes. Nine pathways: Neuroactive ligand-receptor interaction, Tight junction, Rheumatoid arthritis, Transcriptional misregulation in cancers, Focal adhesion, Vascular smooth muscle contraction, Mismatch repair, Other types of O-glycan biosynthesis and Adherens junction were significantly enriched ( $p \leq$ 0.05). Furthermore, pathways such as Platelet activation, Fc gamma R-mediated phagocytosis, Endocytosis, Cytokine-cytokine receptor interaction, and TGF-beta signaling pathway were also enriched $(p>0.05)$. Fc gamma R-mediated phagocytosis and cytokine-cytokine receptor interaction were involved in host immune response to pathogen infection (Figure 3B, Table S8).

\section{Integrated analysis of circRNAs-miRNAs-mRNAs}

CircRNAs could serve as miRNA sponges indirectly regulating gene expression. Therefore, we constructed ceRNA networks based on up-regulated circRNAs, down-regulated miRNAs and up-regulated genes or down-regulated circRNAs, up-regulated miRNAs and down-regulated genes respectively to explore the biological functions of circRNAs during ORFV infection (Figure 4). Interestingly, we found that chi-miR-103-5p_R-7, chi-miR-26b-3p, chi-miR-92a-5p, and chi-miR122-R_1 could bind at least four circRNAs. Novel miRNA PC_3p-10316_124 was shared by both circRNA-186 and circRNA-10457 while chi-miR-2335 and circRNA8386 showed unique binding. The validated circRNA1684 could function as sponge of chi-miR-92a-5p indirectly downregulating eleven genes and the validated circRNA-3127 could bind chi-miR-103-5P downregulating eight genes.

\section{Validation of miRNAs and circRNAs}

The qRT-PCR results showed all six novel miRNAs could be specifically amplified. The expression levels of PC-3p-4306_468, PC-5p-5127_361 and PC-3p-10316_124 were consistent 
247

248

249

250

251

252

253

254

255

256

257

258

259

260

261

262

263

264

265

266

267

268

269

270

271

272

273

274

275

276

277

278

279

280

281

282

283

284

285

286

287

288

289

290

291

with the results of small RNA sequencing while the remaining three miRNAs did not show significant differential expression (Figure S1).

The relative expression levels of nine circRNAs were validated by qRT-PCR using specific divergent primers. The results indicated that circRNA1001, circRNA1684 and circRN3127 were down-regulated while circRNA7880 was up-regulated, which were consistent with the RNA-seq data (Figure 5A). As expected, the results of the agarose gel electrophoresis demonstrated that divergent primers could only amplify circRNAs from cDNA samples, while the convergent primers amplified products from both gDNA and cDNA. (Figure 5B). Back-splicing junctions of circRNAs from cDNA samples were further validated by Sanger sequencing (Figure 5C).

\section{Discussion}

Transcriptional profiling is a powerful tool for researching the host-virus interactions during infection. Recently, researchers performed RNA-seq of sheep oral mucosa in response to Orf virus infection (Jia et al. 2017). They found that multiple differentially expressed genes were enriched in GO terms such as immune response, inflammatory response and apoptosis, indicating that the host could defend virus invasion through immune response and induce cell apoptosis to block viral proliferation. Another study reported alterations of transcriptional profiles in human foreskin fibroblast cells following ORFV infection (Chen et al. 2017). A variety of genes involved in immune response, apoptosis, cell cycle, etc were differentially expressed. These studies provided new insights into the mechanisms of infection by orf virus. Accumulating evidence indicated that circRNAs, a new type of non-coding RNAs, played key roles in diverse diseases ( $\mathrm{Li}$ et al.2015; Tian et al. 2017). Li et al. reported that circular RNA ITCH had inhibitory effect on ESCC by suppressing the Wnt/ $\beta$-catenin pathway. Tian et al. revealed that hsa_circ_0043256 could inhibit cell proliferation and induce apoptosis by acting as a miR-1252 sponge. Although the mechanisms underlying ORFV-host interactions were thoroughly investigated, whether circRNAs were involved in the interactions between ORFV and its host yet remained unknown. Hence, in the current study, we conducted deep circRNA sequencing and small RNA sequencing to identify differentially expressed circRNAs, miRNAs and mRNAs and expected to find the potential circRNA-miRNA-mRNA network existing in interactions between ORFV and its host.

After circRNA sequencing, $\mathrm{R}$ package-psych was used to perform PCA (Principle Component Analysis) statistics investigating the correlations coefficient among the three virusinfected samples and the three mock samples. According to the values of each sample in the first principal component (PC1) and the second principal component (PC2), a two-dimensional coordinate map is made (Figure S2). The results indicated that the three mock samples were close to each other and they were clearly distinct from the three virus-infected samples. There existed a good similarity among samples in the same group and the obvious distinction between the virusinfected samples and the mock samples. Based on the existing criteria for identifying candidate circRNAs, approximately ten thousand circRNAs were detected in uninfected and ORFV-infected GSF cells. Previous studies have also demonstrated that circRNAs are abundant in mammalian cells (Guo et al. 2014; Salzman et al. 2012). However, if we only include the circRNAs detected in at least two of the three replicates in uninfected or ORFV-infected GSF samples, the number of candidate circRNAs decreased to a large extent. There were only 2,935 circRNAs detected in uninfected GSF samples while 2,359 circRNAs were detected in OV samples (Table S9, S10). This would significantly reduce false positives of candidate circRNAs identified in GSF samples or OV samples. Furthermore, we found that $>98 \%$ of circRNAs were ecirRNAs, which differs from Hu's finding in which $86 \%$ of circRNAs were ciRNAs while only $13 \%$ were ecircRNAs and 
292

293

294

295

296

297

298

299

300

301

302

303

304

305

306

307

308

309

310

311

312

313

314

315

316

317

318

319

320

321

322

323

324

325

326

327

$1 \%$ were exon-intron circRNAs derived from the pre-ovulatory ovarian follicles of goats (Tao et al. 2017). These discrepancies further demonstrate that the expression patterns of circRNAs are tissue specific and cell specific.

ORFV infection influenced the circRNA expression profile of the host cells. Compared with the GSF samples, 151 differentially-expressed circRNAs derived from 90 parental genes were identified. Our findings showed that a single gene locus could produce one, two, or several circRNAs through alternative splicing (Table S4). Diverse circRNA isoforms derived from the same cognate linear gene were differentially expressed in ORFV-infected GSF cells, indicating that their parental genes played significant roles in regulating the temporal expression of circRNAs. Fang et al. (Fang et al. 2018) recently reported that circ-Ccnb1 derived from its parental gene CCNB1, a regulator of cell mitosis, had an inhibitory effect on breast cancer cell proliferation and survival. The authors suggested that the biological functions of circRNAs might be closely associated with its parental gene. Next, we performed GO and KEGG analyses for the cognate linear isoforms of the differentially expressed circRNAs to explore the biological functions of circRNAs in response to ORFV infection. In the biological process oncology, regulation of inflammatory response, negative regulation of insulin secretion, positive regulation of cell migration, positive regulation of ubiquitin-protein transferase activity, regulation of ion transmembrane transport were significantly enriched with $p \leq 0.05$ (Figure 3A, Table S7). CircRNA12709, circRNA14794 and circRNA14795, enriched in GO term "regulation of inflammatory response", were different isoforms of their parental gene TNIP (TNFAIP3interacting protein 1) which were up-regulated in OV samples compared to GSF samples during ORFV infection. TNFAIP3 (tumor necrosis factor $\alpha$-induced protein 3 ) also called A20 encoded a ubiquitin-editing protein which was an inhibitor of NF- $\kappa$ B. TNIP1 was shown to play a role in NF$\kappa \mathrm{B}$ inhibition by interacting with A20 (Aya et al. 2010). Pathways such as Tight junction, Rheumatoid arthritis, Transcriptional misregulation in cancers, Focal adhesion, Vascular smooth muscle contraction, Mismatch repair and Other types of O-glycan biosynthesis were significantly enriched $(p \leq 0.05)$. These findings indicated that differential expression of circRNAs may be involved in many biological processes and cellular response to ORFV infection, providing us some valuable clues about the functions of circRNAs. Given that circRNAs could function as miRNA sponges regulating gene expression. We constructed a ceRNA network to explore the potential functions of differentially expressed circRNAs during ORFV infection. For example, circRNA302, circRNA1684, circRNA2565, circRNA4319, circRNA7192, circRNA8828 and circRNA10352 were predicted to sponge chi-miR-92a-5p. Also, circRNA131, circRNA302, circRNA9787, circRNA11661 potentially bound chi-miR-122. These results revealed that a potential ceRNA regulatory network existed in the host-ORFV interaction, although the exact regulatory mechanism requires further investigation.

\section{Conclusion}

In conclusion, we identified 9,979 and 10,844 circRNAs in GSF cells before and after ORFV infection. A total of 151 circRNAs (59 circRNAs up-regulated and 92 circRNAs down-regulated) 341 mRNAs, and 56 miRNAs were differentially expressed following ORFV infection. Four circRNAs: circRNA1001, circRNA1684, circRNA3127 and circRNA7880 were validated by qRT-PCR and Sanger sequencing. Host genes of differentially expressed circRNAs were significantly enriched in many biological processes including regulation of inflammatory response, positive regulation of cell migration, and regulation of ion transmembrane transport. A potential circRNA-miRNA-mRNA regulatory network exists during ORFV infection. Our study 
337

338

339

340

341

342

343

is the first to present the expression profiles of circRNAs in GSF cells in response to ORFV infection and may provide new insights into the mechanism underlying ORFV pathogenesis.

\section{Acknowledgments}

We thank Tamsin Sheen, PhD, from Liwen Bianji, Edanz Editing China (www.liwenbianji.cn/ac), for editing the English text of a draft of this manuscript.

\section{References}

Agarwal V, Bell GW, Nam JW, and Bartel DP. 2015. Predicting effective microRNA target sites in mammalian mRNAs. eLife, 4 : e05005.

Aya K, Satoshi I, Hiroshi F, Taichi H, Daisuke G, Isao M, Makio K, Jun O, Graham RR, and Kunio M. 2010. Association of TNFAIP3 interacting protein 1, TNIP1 with systemic lupus erythematosus in a Japanese population: a case-control association study[J]. Arthritis research \& therapy, 12(5): R174.

Barrett SP, and Salzman J. 2016. Circular RNAs: analysis, expression and potential functions. Development 143:1838.

Barrett SP, Wang PL, and Salzman J. 2015. Circular RNA biogenesis can proceed through an exon-containing lariat precursor. eLife,4: e07540.

Beißbarth T, and Speed TP. 2004. GOstat: find statistically overrepresented Gene Ontologies within a group of genes. Bioinformatics 20:1464.

Betel D, Wilson M, Gabow A, Marks DS, and Sander C. 2008. The microRNA.org resource: targets and expression. Nucleic Acids Research 36:149-153.

Chen D, Long M, Xiao B, Xiong Y, Chen H, Chen Y, Kuang Z, Li M, Wu Y, and Rock DL. 2017. Transcriptomic profiles of human foreskin fibroblast cells in response to orf virus. Oncotarget 8:58668-58685.

Chi X, Zeng X, Li W, Hao W, Li M, Huang X, Huang Y, Rock DL, Luo S, and Wang S. 2015. Genome analysis of orf virus isolates from goats in the Fujian Province of southern China. Frontiers in Microbiology 6:1135.

Consortium GO. 2004. The Gene Ontology (GO) database and informatics resource. Nucleic Acids Res 32:D258-261.

Delhon G, Tulman ER, Afonso CL, Lu Z, De 1C-BA, Lehmkuhl HD, Piccone ME, Kutish GF, and Rock DL. 2004. Genomes of the parapoxviruses ORF virus and bovine papular stomatitis virus. Journal of Virology 78:168.

Fang L, Du WW, Lyu J, Dong J, Zhang C, Yang W, He A, Kwok Y, Ma J, and Wu N. 2018. Enhanced breast cancer progression by mutant p53 is inhibited by the circular RNA circCcnb1. Cell Death \& Differentiation.

Frazee AC, Pertea G, Jaffe AE, Langmead B, Salzberg SL, and Leek JT. 2015. Ballgown bridges the gap between transcriptome assembly and expression analysis. Nature Biotechnology 33:243.

Friederichs S, Krebs S, Blum H, Wolf E, Lang H, Buttlar HV, and Büttner M. 2014. Comparative and retrospective molecular analysis of Parapoxvirus (PPV) isolates. Virus Research 181:11-21.

Guo JU, Agarwal V, Guo H, and Bartel DP. 2014. Expanded identification and characterization of mammalian circular RNAs. Genome Biology 15:409.

Hansen TB, Jensen TI, Clausen BH, Bramsen JB, Finsen B, Damgaard CK, and Kjems J. 2013. 
Natural RNA circles function as efficient microRNA sponges. Nature 495:384-388.

He J, Xie Q, Xu H, Li J, and Li Y. 2017a. Circular RNAs and cancer. Cancer Letters 396:138.

He L, Zhang A, Xiong L, Li Y, Huang R, Liao L, Zhu Z, and Wang AY. 2017b. Deep Circular RNA Sequencing Provides Insights into the Mechanism Underlying Grass Carp Reovirus Infection. International Journal of Molecular Sciences 18:1977.

Jia H, Zhan L, Wang X, He X, Chen G, Zhang Y, Feng Y, Wei Y, Zhang Y, Jing Z. Transcriptome analysis of sheep oral mucosa response to Orf virus infection. Plos One, 12(10):e0186681.

Jeck WR, Sorrentino JA, Wang K, Slevin MK, Burd CE, Liu J, Marzluff WF, and Sharpless NE. 2013. Circular RNAs are abundant, conserved, and associated with ALU repeats. Rna-a Publication of the Rna Society 19:141-157.

Kanehisa M, Furumichi M, Tanabe M, Sato Y, and Morishima K. 2017. KEGG: new perspectives on genomes, pathways, diseases and drugs. Nucleic Acids Research 45:D353-D361.

Kanehisa M, Goto S, Sato Y, Furumichi M, and Tanabe M. 2012. KEGG for integration and interpretation of large-scale molecular data sets.

Kim D, Pertea G, Trapnell C, Pimentel H, Kelley R, and Salzberg SL. 2013. TopHat2: accurate alignment of transcriptomes in the presence of insertions, deletions and gene fusions. Genome Biology 14:R36.

Kim D, and Salzberg SL. 2011. TopHat-Fusion: an algorithm for discovery of novel fusion transcripts. Genome Biology, 12,8:R72.

Kumar N, Wadhwa A, Chaubey KK, Singh SV, Gupta S, Sharma S, Sharma DK, Singh MK, and Mishra AK. 2014. Isolation and phylogenetic analysis of an orf virus from sheep in Makhdoom, India. Virus Genes 48:312-319.

Langmead B, and Salzberg SL. 2012. Fast gapped-read alignment with Bowtie 2. Nature Methods 9:357-359.

Li F, Zhang L, Li W, Deng J, Zheng J, An M, Lu J, Zhou Y. Circular RNA ITCH has inhibitory effect on ESCC by suppressing the Wnt/ $\beta$-catenin pathway. 2015.Oncotarget 6(8):6001.

Martin M. 2011. Cutadapt removes adapter sequences from high-throughput sequencing reads. Embnet Journal 17.

Martins M, Joshi LR, Rodrigues FS, Anziliero D, Frandoloso R, Kutish GF, Rock DL, Weiblen R, Flores EF, and Diel DG. 2017. Immunogenicity of ORFV-based vectors expressing the rabies virus glycoprotein in livestock species. Virology 511:229-239.

Memczak S, Jens M, Elefsinioti A, Torti F, Krueger J, Rybak A, Maier L, Mackowiak SD, Gregersen LH, Munschauer M, Loewer A, Ziebold U, Landthaler M, Kocks C, le Noble F, and Rajewsky N. 2013. Circular RNAs are a large class of animal RNAs with regulatory potency. Nature 495:333-338. 10.1038/nature11928

Peng F, Chen Z, Zheng SY, Li HM, Du J, and Zhang JZ. 2016. A Case of Orf Identified by Transmission Electron Microscopy. Chin Med J (Engl) 129:108.

Pertea M, Pertea GM, Antonescu CM, Chang TC, Mendell JT, and Salzberg SL. 2015. StringTie enables improved reconstruction of a transcriptome from RNA-seq reads. Nature Biotechnology 33:290-295.

Qu S, Yang X, Li X, Wang J, Gao Y, Shang R, Sun W, Dou K, and Li H. 2015. Circular RNA: A new star of noncoding RNAs. Cancer Letters 365:141-148.

Rajkomar V, Hannah M, Coulson IH, and Owen CM. 2016. A case of human to human transmission of orf between mother and child. Clinical \& Experimental Dermatology 41:60-63.

Robinson MD, Mccarthy DJ, and Smyth GK. 2014. edgeR: a Bioconductor package for differential 
expression analysis of digital gene expression data.

Salzman J, Chen RE, Olsen MN, Wang PL, and Brown PO. 2013. Cell-Type Specific Features of Circular RNA Expression. Plos Genetics 9:e1003777.

Salzman J, Gawad C, Wang PL, Lacayo N, and Brown PO. 2012. Circular RNAs Are the Predominant Transcript Isoform from Hundreds of Human Genes in Diverse Cell Types. Plos One 7:e30733.

Shannon P, Markiel A, Ozier O, Baliga NS, Wang JT, Ramage D, Amin N, Schwikowski B, and Ideker T. 2003. Cytoscape: A Software Environment for Integrated Models of Biomolecular Interaction Networks. Genome Research 13:2498.

Shi J, Hu N, Li J, Zeng Z, Mo L, Sun J, Wu M, and Hu Y. 2017. Unique expression signatures of circular RNAs in response to DNA tumor virus SV40 infection. Oncotarget 8:9860998622.

Suzuki H, Zuo Y, Wang J, Zhang MQ, Malhotra A, and Mayeda A. 2006. Characterization of RNase R-digested cellular RNA source that consists of lariat and circular RNAs from premRNA splicing. Nucleic Acids Res 34:e63. 10.1093/nar/gk1151

Szabo L, Morey R, Palpant NJ, Wang PL, Afari N, Jiang C, Parast MM, Murry CE, Laurent LC, and Salzman J. 2015. Statistically based splicing detection reveals neural enrichment and tissue-specific induction of circular RNA during human fetal development. Genome Biology 16:1-26.

Tao H, Xiong Q, Zhang F, Zhang N, Liu Y, Suo X, Li X, Yang Q, and Chen M. 2017. Circular RNA profiling reveals chi_circ_0008219 function as microRNA sponges in pre-ovulatory ovarian follicles of goats (Capra hircus). Genomics, 110(4): 257-266.

Tian F, Yu CT, Ye WD, and Wang Q. 2017. Cinnamaldehyde induces cell apoptosis mediated by a novel circular RNA hsa_circ_0043256 in non-small cell lung cancer. Biochem Biophys Res Commun 493:1260.

Zhang K, Liu Y, Kong H, Shang Y, and Liu X. 2014a. Comparison and phylogenetic analysis based on the B2L gene of orf virus from goats and sheep in China during 2009-2011. Archives of Virology 159:1475-1479.

Zhang XO, Dong R, Zhang Y, Zhang JL, Luo Z, Zhang J, Chen LL, and Yang L. 2016. Diverse alternative back-splicing and alternative splicing landscape of circular RNAs. Genome Research 26:1277-1287.

Zhang XO, Wang HB, Zhang Y, Lu X, Chen LL, and Yang L. 2014b. Complementary sequencemediated exon circularization. Cell 159:134-147.

Zhao K, Song D, He W, Lu H, Zhang B, Li C, Chen K, and Gao F. 2010. Identification and phylogenetic analysis of an Orf virus isolated from an outbreak in sheep in the Jilin province of China. Vet Microbiol 142:408-415. 


\section{Table 1 (on next page)}

List of convergent and divergent primers used for circRNAs qRT-PCR validation. 


\begin{tabular}{|c|c|c|}
\hline Primers & Sequences (5'-3') & Size (bp) \\
\hline GAPDH CON-F & AGCCGTAACTTCTGTGCTGT & \multirow{2}{*}{234} \\
\hline GAPDH CON-R & TTCCCGTTCTCTGCCTTGAC & \\
\hline GAPDH DIV-F & ATGGTCCACATGGCCTCC & \\
\hline GAPDH DIV-R & CATCTTGTCTCAGGGATGC & \\
\hline circRNA1001 CON-F & CCACTCAGTTCCCTGCTGAT & \multirow{2}{*}{82} \\
\hline circRNA1001 CON-R & TCTTTACTTTGTGGCTGGCTC & \\
\hline circRNA1001 DIV-F & TAGACAGCTCTGACAGCATGG & \multirow{2}{*}{101} \\
\hline circRNA1001 DIV-R & TGCCACATGACTCATTAATTTC & \\
\hline circRNA1684 CON-F & GGAGTCAACCTCACCACTGA & \multirow{2}{*}{71} \\
\hline circRNA1684 CON-R & CCCCGGTCATAGCACACAA & \\
\hline circRNA1684 DIV-F & CGATTACTCCATGTACCAGGCA & \multirow{2}{*}{155} \\
\hline circRNA1684 DIV-R & GCACACAAACCTGTAATCCTGG & \\
\hline circRNA3127 CON-F & AGGACCCTCATCCCTCGTTA & \multirow{2}{*}{114} \\
\hline circRNA3127 CON-R & GTCCACGGTGATGGATGAGTT & \\
\hline circRNA3127 DIV-F & TCACCCTCAACTACCTCAGGCT & \multirow{2}{*}{78} \\
\hline circRNA3127 DIV-R & GTCACCССТCСТTCAAACACAG & \\
\hline circRNA7880 CON-F & AAAAGAAGCCGTCTCGGACA & \multirow{2}{*}{132} \\
\hline circRNA7880 CON-R & CCAGACGTTTTCTGGGGCTA & \\
\hline circRNA7880 DIV-F & AATCAGATAGCCACCATCTTG & \multirow{2}{*}{85} \\
\hline circRNA7880 DIV-R & TGTAGCCTGTGACTGGGAAC & \\
\hline circRNA998 DIV-F & GACGACCTGATGGATTATCACC & \multirow{2}{*}{89} \\
\hline circRNA998 DIV-R & TGCCATAATCTTGTTGGAATCA & \\
\hline circRNA1000 DIV-F & TCGGAAACAACTGAACTTATGA & \multirow{2}{*}{125} \\
\hline circRNA1000 DIV-R & TGTTCTTCACTTATACCCTCTGG & \\
\hline circRNA4287 DIV-F & GTGTGAAAATAACGTGAAGGAA & \multirow{2}{*}{102} \\
\hline circRNA4287 DIV-R & СТTCTAАTTTCCTCACTCTCAGA & \\
\hline circRNA5112 DIV-F & GGCTAAGCAATTCTCGGTTGG & \multirow{2}{*}{77} \\
\hline circRNA5112 DIV-R & TTGTAGCCTGTGACTGGGAACG & \\
\hline circRNA8565 DIV-F & GCTACTTCCAGCTGCAGATGTG & \multirow{2}{*}{137} \\
\hline circRNA8565 DIV-R & ACACTGAGAACTTCAGGAACGC & \\
\hline
\end{tabular}

2

3 Table 1: List of convergent and divergent primers used for circRNAs qRT-PCR validation. 4 
Table 2 (on next page)

Summary of circRNA sequencing data. 
1

\begin{tabular}{|c|c|c|c|c|c|c|}
\hline Sample & GSF-1 & GSF-2 & GSF-3 & $\mathrm{OV}-1$ & $\mathrm{OV}-2$ & OV-3 \\
\hline Raw reads & $84,146,788$ & $89,058,270$ & $85,890,172$ & $89,408,452$ & $93,833,004$ & $97,376,392$ \\
\hline Valid reads & $81,678,856$ & $86,169,216$ & $83,244,598$ & $86,920,674$ & $91,594,088$ & $95,061,748$ \\
\hline Mapped reads (linear) & $70466868(86.27 \%)$ & $72820127(84.51 \%)$ & $70690768(84.92 \%)$ & $75616664(87.00 \%)$ & $79582058(86.89 \%)$ & $82061025(86.32 \%)$ \\
\hline Unmapped reads & $11211988(13.73 \%)$ & $13349089(15.49 \%)$ & $12553830(15.08 \%)$ & $11304010(13.00 \%)$ & $12012030(13.11 \%)$ & $13000723(13.68 \%)$ \\
\hline Back-spliced junctions reads & $799766(0.98 \%)$ & $1133133(1.32 \%)$ & $905843(1.09 \%)$ & $874057(1.01 \%)$ & $766106(0.84 \%)$ & $889334(0.94 \%)$ \\
\hline Reads mapped to Orf virus & $41(0.00 \%)$ & $28(0.00 \%)$ & $17(0.00 \%)$ & $125619(1.11 \%)$ & $165878(1.38 \%)$ & $157779(1.21 \%)$ \\
\hline Q30 & $93.38 \%$ & $92.27 \%$ & $93.08 \%$ & $94.02 \%$ & $93.80 \%$ & $93.73 \%$ \\
\hline
\end{tabular}

3 Table 2. Summary of circRNA sequencing data 


\section{Figure 1}

Properties of circRNAs in ORFV-infected and uninfected GSF samples.

(A) Venn diagram showing the number of circRNAs either shared between or uniquely expressed in the GSF samples and ORFV-infected samples. (B) Pie chart showing the prevalence of the different circRNA types. ciRNAs from introns are represented by the orange section, while the blue region indicates ecircRNAs from exons. (C) Distribution of circRNAs among the chromosomes of the goat reference genome. (D) Lengths of the circRNAs from the GSF and ORFV-infected samples. (E) Number of circRNA isoforms derived from the same gene. 

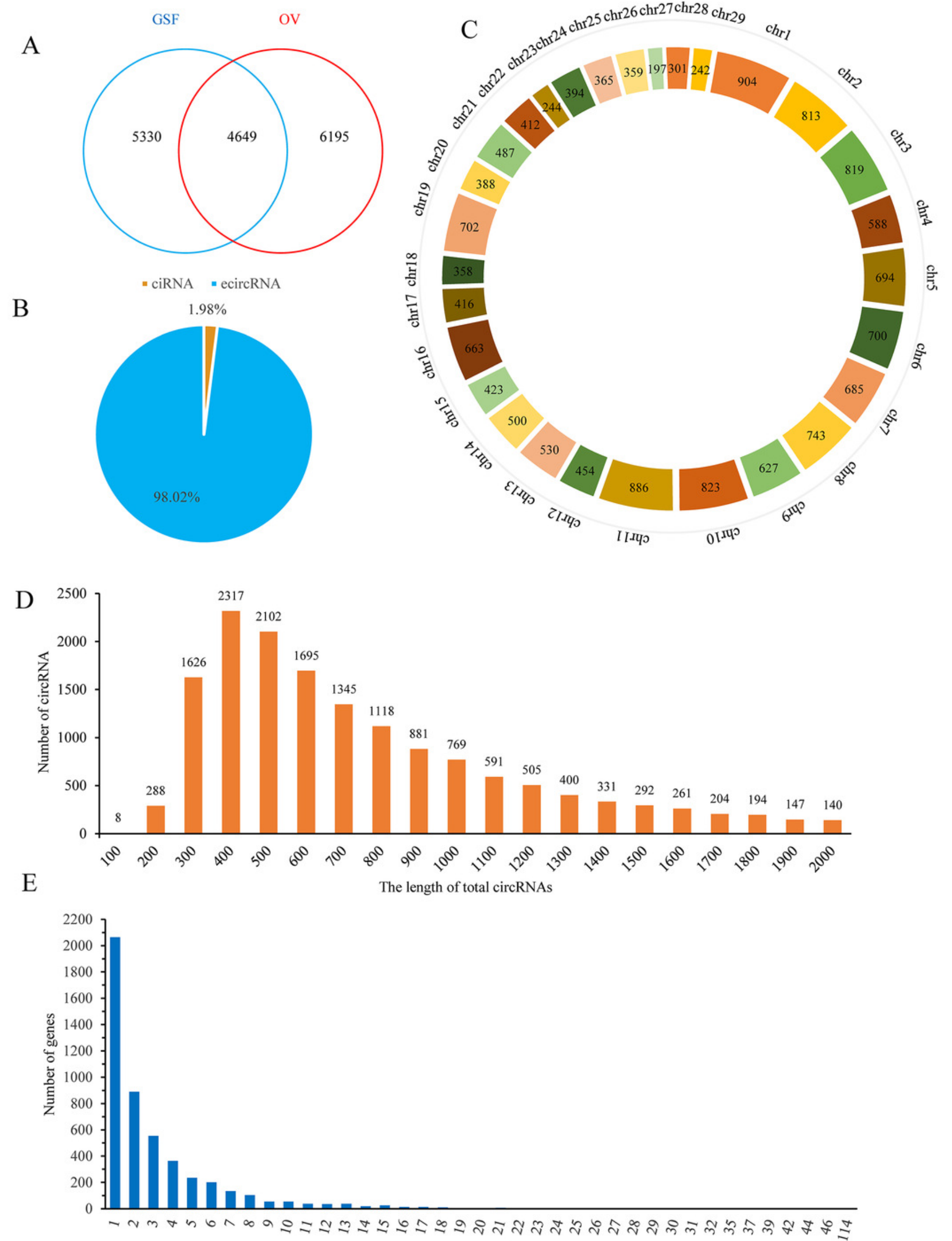


\section{Figure 2}

Differentially expressed circRNAs, mRNAs, miRNAs in ORFV-infected samples compared with GSF samples.

(A) Bar charts showing the number of differentially expressed circRNAs, mRNAs, miRNAs, respectively. Red bars, up-regulated RNAs; green bars, down-regulated RNAs. (B) Heatmap of differentially expressed circRNAs. (C) Heatmap of differentially expressed miRNAs. (D) Heatmap of differentially expressed mRNAs. Color from green to red; the deeper the color, the higher the expression. (E) Volcano plot of differentially expressed mRNAs. Vertical lines correspond to 2-fold changes in up-regulation and down-regulation. Horizontal line represents $p$ value 0.05 . Red points refer to up-regulated mRNAs; Green points refer to down-regulated mRNAs; Blue points refer to mRNAs with no significant difference. 
A
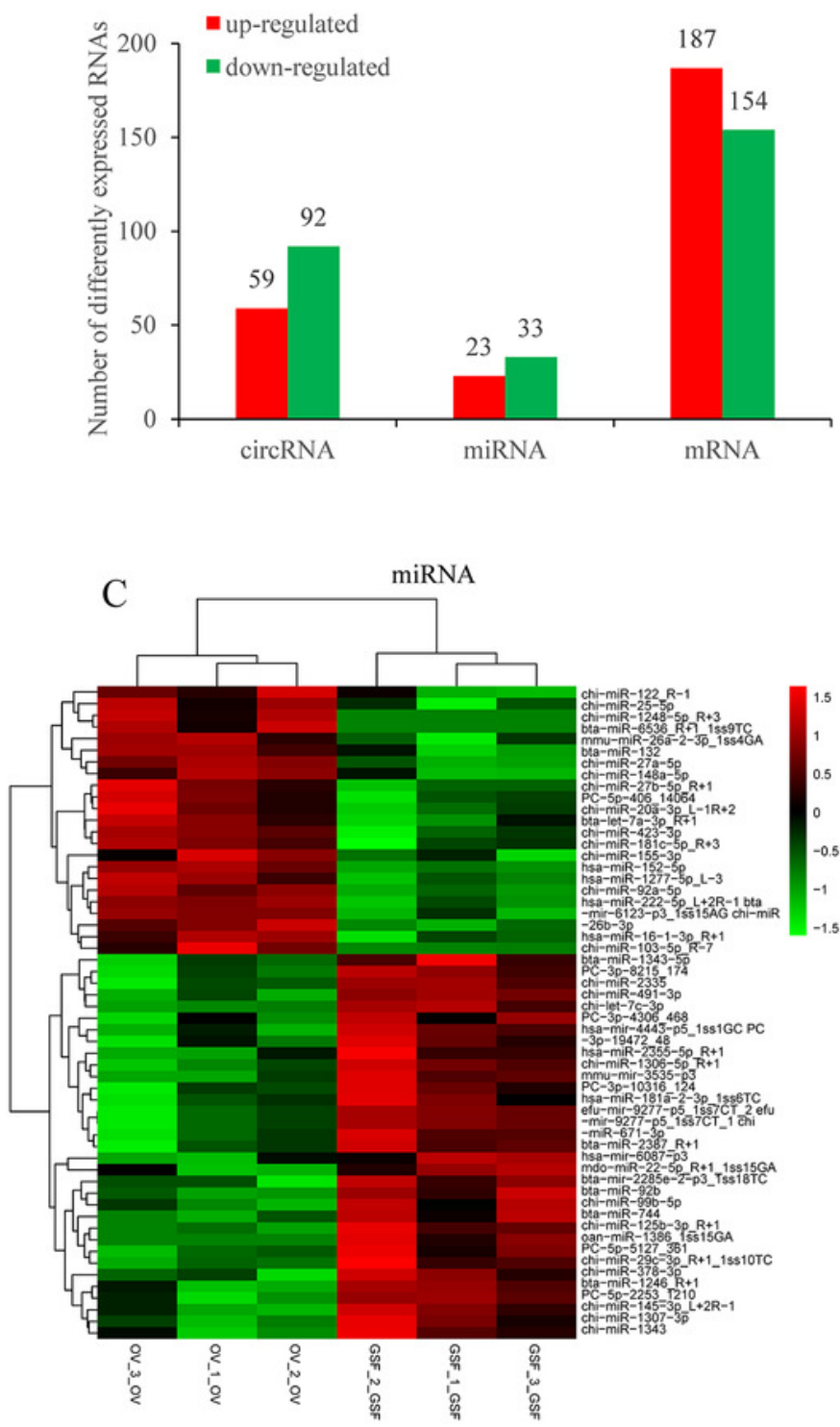

E

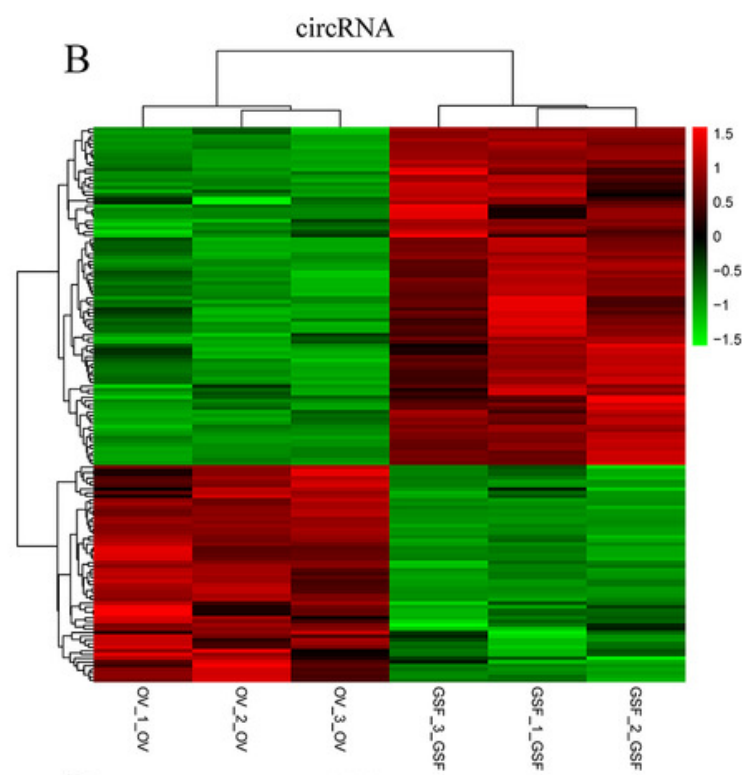

D

mRNA

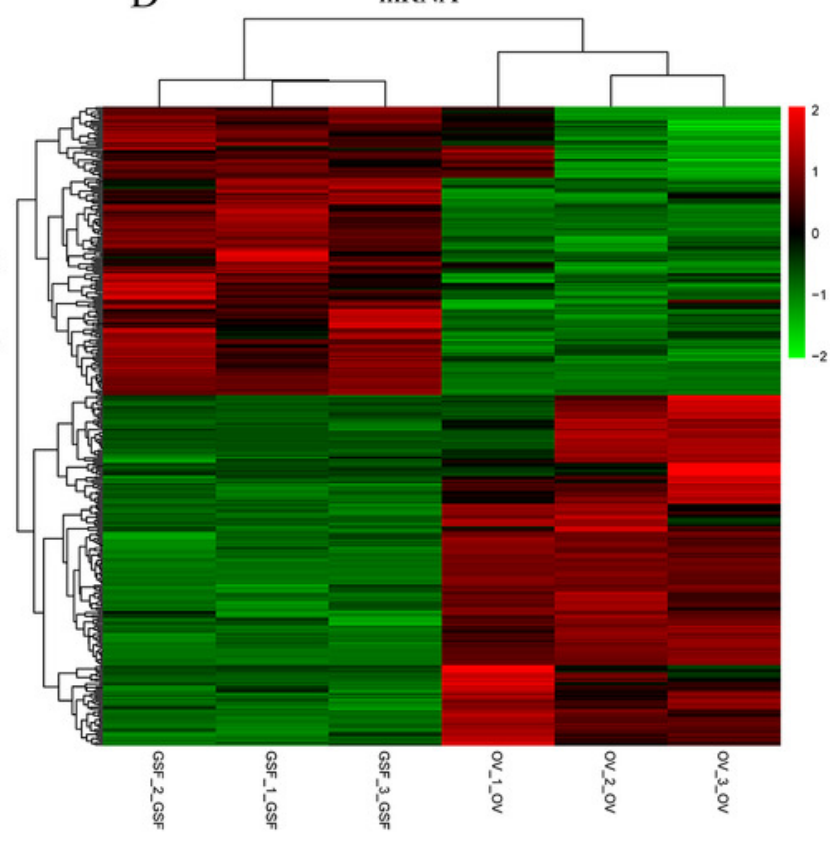

- no difference

- up-regulated

- down-regulated 


\section{Figure 3}

GO and KEGG analyses for host genes of differentially expressed circRNAs.

(A) GO enrichment analysis for host genes of differentially expressed circRNAs. Top 20 GO terms $(p \leq 0.05)$ in biological processes, cellular components and molecular functions are presented. Left Y-axis represents the absolute gene counts enriched in the term; right Y-axis represents \% of the genes enriched in the GO term compared to the total number of genes enriched in top $20 \mathrm{GO}$ terms in three ontologies. (B) KEGG pathway enrichment analysis for host genes of differentially expressed circRNAs. Y-axis represents pathways; X-axis represents rich factor; (rich factor equals the ratio between the host genes of differentially expressed circRNAs and all annotated genes enriched in the pathway); The color and size of each bubble represent enrichment significance and the number of genes enriched in a pathway, respectively.

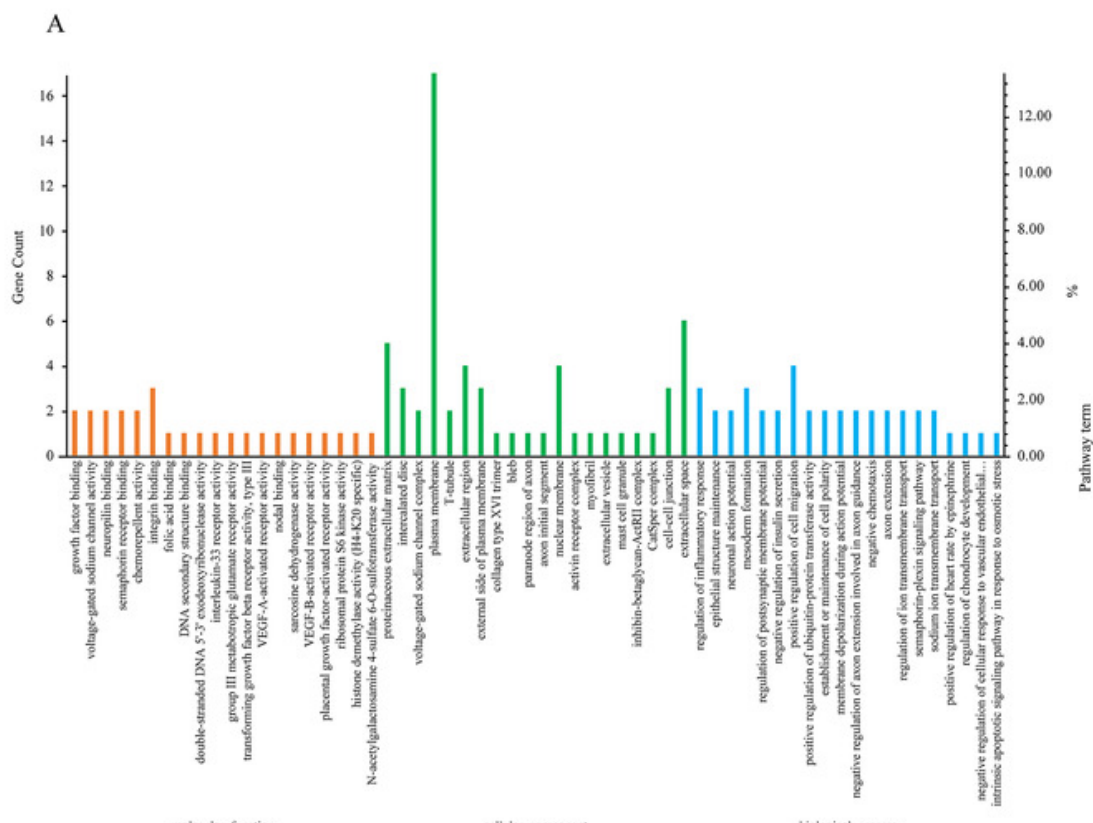

B

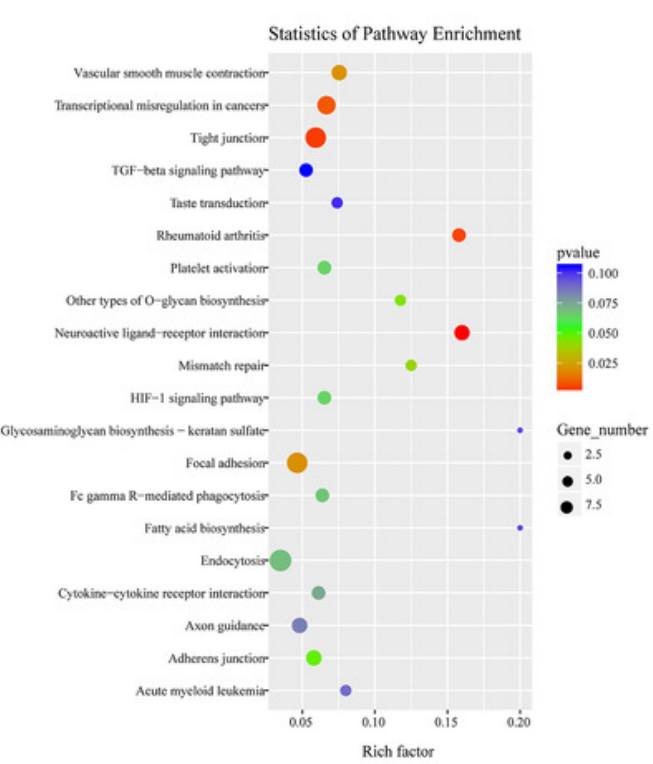




\section{Figure 4}

A ceRNA network.

A ceRNA network based on up-regulated circRNAs, down-regulated miRNAs and up-regulated genes or down-regulated circRNAs, up-regulated miRNAs and down-regulated genes. Red and green represent up- and down-regulation, respectively. Round, Rectangle and Arrow represent circRNAs, miRNAs, and mRNAs, respectively. 

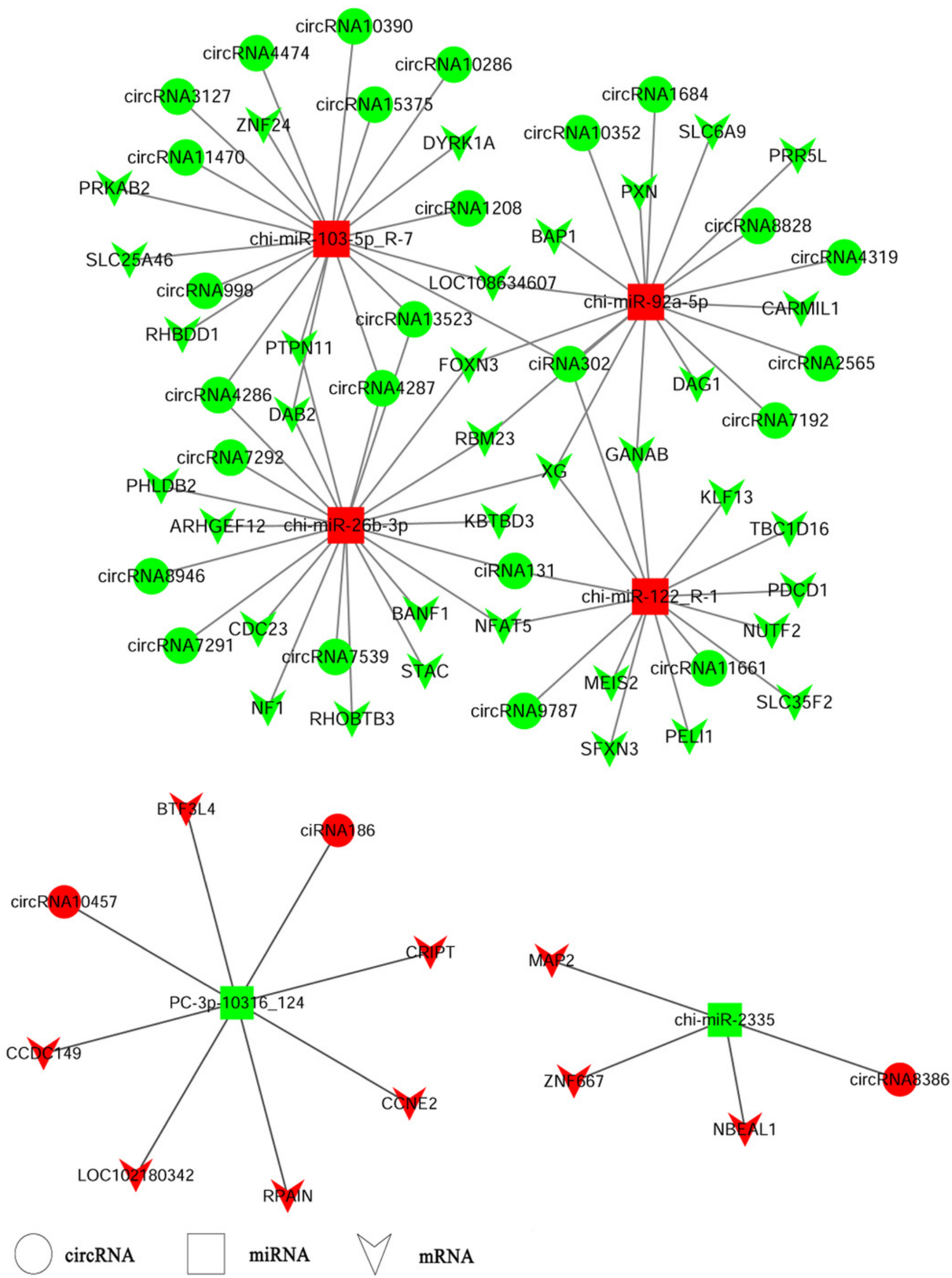


\section{Figure 5}

Validation of circRNAs by qRT-PCR and Sanger sequencing.

(A) Validation of differentially expressed circRNAs using qRT-PCR. Data from qRT-PCR assays are the means of three independent replicates, with error bars representing SD. (B) The PCR amplification by divergent and convergent primers in genomic DNA and CDNA samples. The red asterisks represent the band of circRNAs from cDNA samples. The linear GADPH gene serves as an internal control. (C) Sanger sequencing confirmation of back-splicing junctions of circRNAs. Back-splicing sites are indicated with black arrows. 
A
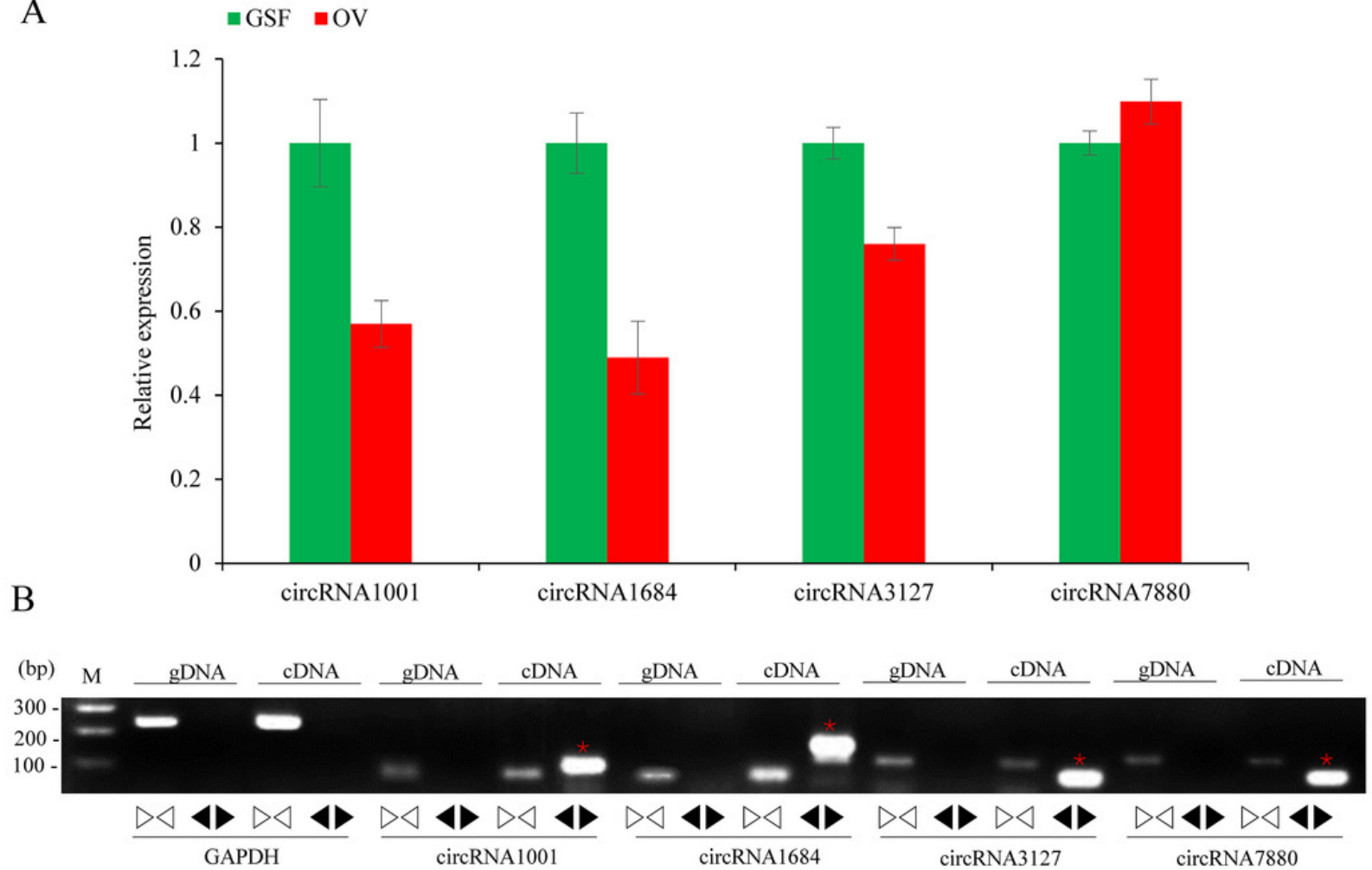

$\mathrm{C}$

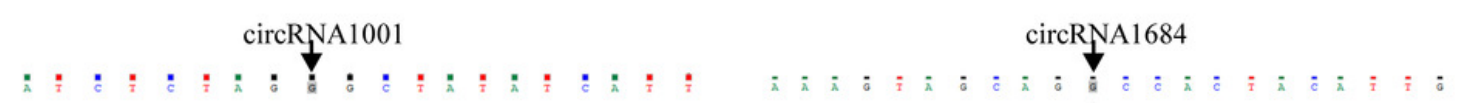

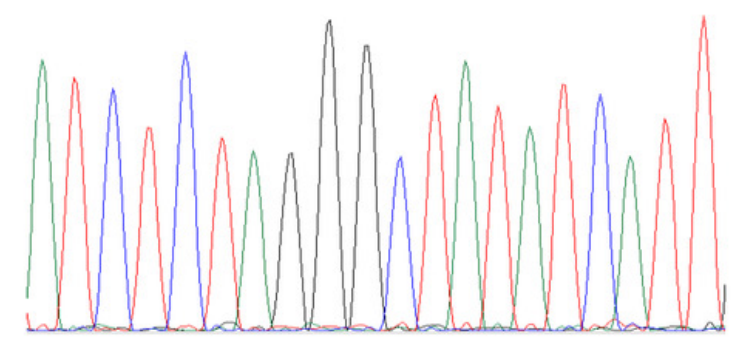

circRNA3127
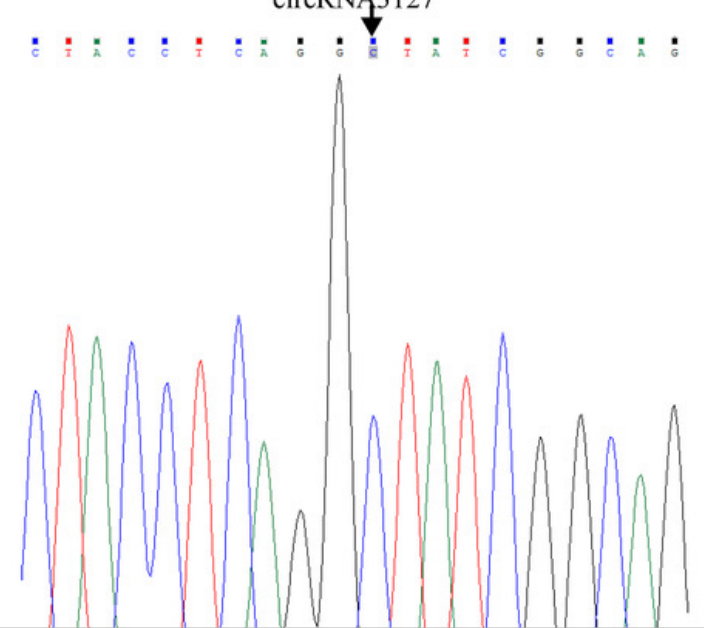

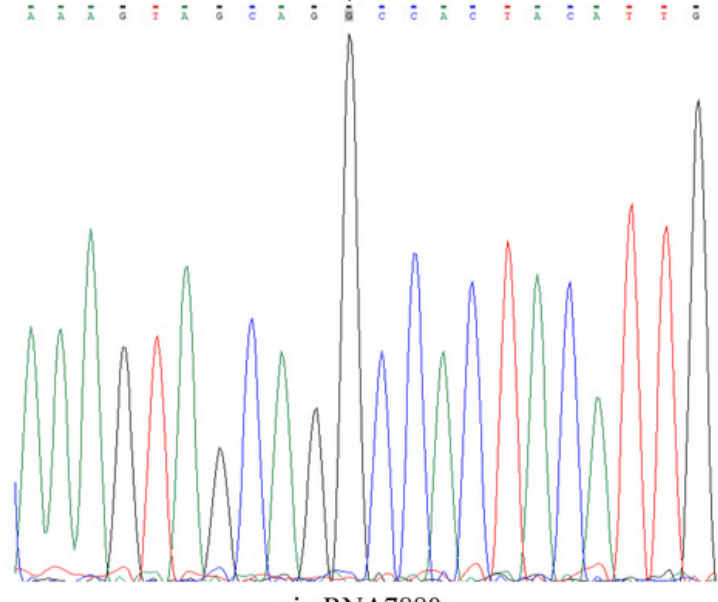

circRNA7880

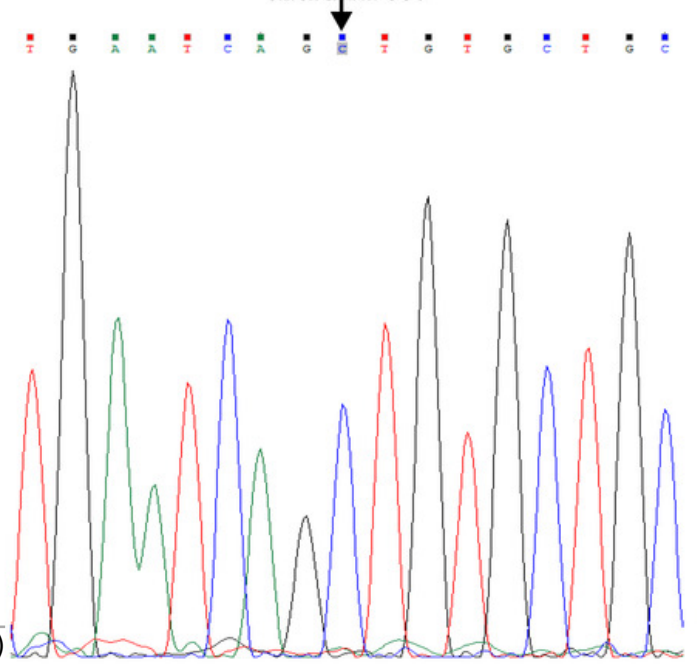

